\title{
A Simple and High-yield Synthesis of Hexadecyl Ferulate and Its In Vitro Antioxidant Potential
}

\author{
Jessica Nadal ${ }^{1 *}$, Flávia de B. Pedroso ${ }^{1}$, Bruno Rodrigo Minozzo ${ }^{1}$, Priscilla Salles de Brito ${ }^{1}$, \\ Paulo Vitor Farago ${ }^{1}$, José Carlos Rebuglio Vellosa ${ }^{2}$, Edmar Miyoshi ${ }^{1}$ \\ ${ }^{1}$ Universidade Estadual de Ponta Grossa - Departamento de Ciências Farmacêuticas, Ponta Grossa, Paraná, \\ Brasil. ${ }^{2}$ Universidade Estadual de Ponta Grossa - Departamento de Análises Clínicas e Toxicológicas
}

\begin{abstract}
Ferulic acid (FA) is a phenolic compound with well-known antioxidant potential that can be used as a promising anti-inflammatory and anti-cancer molecule. Furthermore, it has been reported to have neuroprotective activity. One of the main problems, which limit its clinical use, is its low bioavailability when administered orally. This limitation can be circumvented by changes in their structure and/or for preparing lipid-based formulations. The aim of this study was to synthesize a derivative of $F A$, the hexadecyl ferulate (HF). This compound would be more susceptible to pass through blood-brain barrier $(B B B)$ due to its lipophilic character. The HF was obtained by Steglich esterification and yielded $76.77 \pm 1.35 \%$. Its structural characterization was performed by spectroscopic methods of Fourier-transformed infrared spectroscopy (FTIR) and nuclear magnetic resonance (NMR). FTIR spectrum of HF presented two typical bands of ester group, a $C=O$ ester stretching band at $1725 \mathrm{~cm}^{-1}$ and $a C-O$ stretching band at $1159 \mathrm{~cm}^{-1}$. The ${ }^{1} \mathrm{H}$ and ${ }^{13} \mathrm{C}$ spectral data confirmed the chemical structure of HF. Regarding the ${ }^{13} \mathrm{C} N M R$ spectrum, HF showed a chemical shift at $\delta 167.39 \mathrm{ppm}$ which corresponded to the carbonyl carbon of the ester group. Concerning the in vitro antioxidant potential, HF had equivalent or improved scavenger activity than FA leading to IC50 values of $0.083 \pm 0.009$ nmol. $\mathrm{mL}^{-1}$ and $0.027 \pm 0.002$ nmol.mL $\mathrm{L}^{-1}$ in DPPH radical scavenging and ABTS radical cation decolorization assays, respectively. Further studies are required in order to investigate the antioxidant effect of HF in biological media.
\end{abstract}

Keywords: ABTS•+ assay, DPPH• assay, ferulic acid derivative, scavenger activity, Steglich esterification.

\footnotetext{
*Author for correspondence: jessicabem@hotmail.com
} 


\section{INTRODUCTION}

Ferulic acid (FA; $\mathrm{C}_{10} \mathrm{H}_{10} \mathrm{O}_{4}$; MW: 194.18 Da) or (E)-3-(4-hydroxy-3-methoxyphenyl)prop-2-enoic acid is an organic compound widely available in plants which has low toxicity. It is biosynthesized from shikimate pathway via phenylalanine or Ltyrosine $^{1}$. In nature, FA is usually found as component of plant cell walls offering linkage with lignin; in arabinoxylans, FA is esterified to the C-5 of $\alpha$ larabinofuranose; in pectins, it is esterified to the $\mathrm{C}-2$ of $\alpha 1 \rightarrow 5$-linked arabinofuranose or to the C-6 of $\beta 1 \rightarrow 4$-linked galactopyranose, while in xyloglucans, it is found attached to the $\mathrm{C}-4$ of $\alpha$-D-xylopyranose ${ }^{2,3}$.

FA exhibits a wide range of therapeutic effects against various diseases such as cancer, diabetes, and cardiovascular and neurodegenerative disorders ${ }^{4}$. A wide spectrum of beneficial activities for human health has been proposed for this phenolic compound, at least in part because of its strong antioxidant activity. Considering the effect of FA in central nervous system (CNS), it can attenuate neuronal cell death due to uptake of oxidized low-density lipoprotein, which is generated by reactive oxygen species $(\mathrm{ROS})^{5}$. Due to its phenolic nucleus and an extended side-chain conjugation, it readily forms a resonance-stabilized phenoxyl radical, which accounts for its potent antioxidant effect ${ }^{6}$.

In spite of some papers devoted to obtain possible medicines containing FA, the unfavorable pharmacokinetics, which reduces the bioavailability of FA after oral administration and the restricted number of clinical studies carried out with the purpose of proving FA efficacy and safety ${ }^{7}$, are the main aspects that limit its use as an antioxidant drug in humans. In order to avoid these gaps, changes in its structure and by the preparation of lipid-based formulations have been proposed ${ }^{8}$. However, these problems are more complex when a brain targeting drug delivery system is intended to be achieved. However, there has hitherto been observed a restricted evidence for in vivo permeation of the blood-brain barrier (BBB) by FA. Based upon in vitro results, FA crosses the BBB, but exhibits a lag time ${ }^{9}$.

Many drugs, when employed with no chemical or physicochemical modification, result in reduced pharmacological effects on the CNS due to the difficulty in crossing the BBB. For circumventing this limitation, lipophilization of molecules is an attractive way for increasing the lipophilic properties of a drug. In other words, an active substance is modified by masking polar groups with nonpolar groups, thereby converting a more water-soluble substance to a lipophilic drug ${ }^{10}$.

To address this problem, there are few literature reports devoted to the synthesis of lipophilic derivatives from FA. An intermediate vinyl ferulate was first chemically produced by the enzymatic synthesis and subsequently esterified with phytosterols through alcoholysis with Candida rugosa as a catalyst ${ }^{6}$. Similarly, an optimized synthesis of novel prenyl ferulate was performed by feruloyl esterases from Myceliophthora thermophila in microemulsions ${ }^{3}$. A solvent-free and reduced pressure evaporation system was developed for lipase-catalyzed synthesis of 2-ethylhexyl ferulate from FA and 2-ethylhexanol ${ }^{11}$. Sandoval and co-authors $(2015)^{12}$ synthesized monoesters were further acylated with vinyl esters in order to obtain FA diesters with significantly increased hydrophobicity, which could benefit their performance in food, pharmaceutical, and cosmetic applications.

In this study, we propose to synthesize a monoester by the Steglich esterification ${ }^{13}$, which is a simple and high-yield reaction in order to obtain hexadecyl ferulate (HF), a more lipophilic compound from FA. This derivative substance could keep the scavenging activity of FA at the same time it could present a promising improved behavior in biological systems as CNS. To the best of our knowledge, esterification of FA to its derivative HF has not yet been reported in the literature. In addition, the goal 
of this paper was to perform a complete characterization of this ester in order to investigate its antioxidant activity by some in vitro scavenging models.

\section{MATERIALS AND METHODS}

\section{Materials}

Ferulic acid $99.80 \%$ (FA) was purchased of Suzhou Leader Chemical Co. Ltda. (Suzhou, China). Cetyl alcohol 99\% (CA), N,N'-dicyclohexylcarbodiimide (DCC, 99\%), 4-dimethylaminopyridine (DMAP, $\geq 99 \%$ ) and quercetin $(\geq 95 \%$ ) were obtained from Sigma-Aldrich (St. Louis, MO, USA). Water was purified in a Milli-Q Plus water purification system (Millipore, Bedford, MA, USA). All others reagents and solvents were of analytical grade and were used as received.

\section{METHODS}

\section{Synthesis of hexadecyl ferulate}

As a chemical strategy for synthesizing the FA ester, CA, a long alkyl chain (16 carbons) alcohol, was used. The reaction between FA (1) and CA (2) was developed using 1,3-dicyclohexylcarbodiimide (DCC) (3) as a dehydrating agent and coupling reagent in tetrahydrofuran (THF) (4) and 4-dimethylaminopyridine (DMAP) (5) as a catalyst, based on the paper previously published by Neises and Steglich $(1978)^{13}$. The Steglich esterification was used as a mild reaction consisting of using DCC/DMAP system in order to obtain the final ester (HF) (6) and the by-product dicyclohexylurea (DCU) (7) (Figure 1).

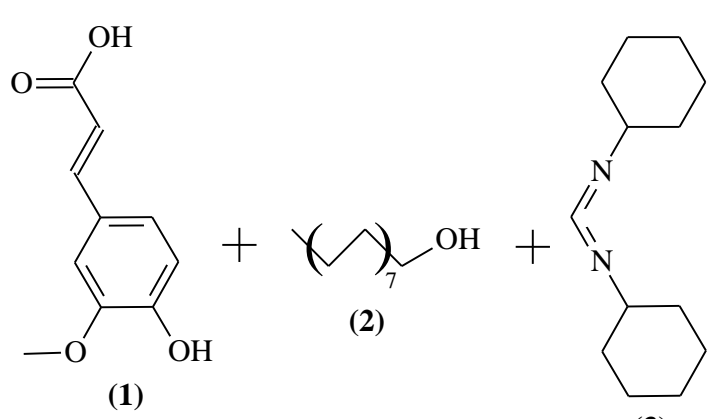

(3)

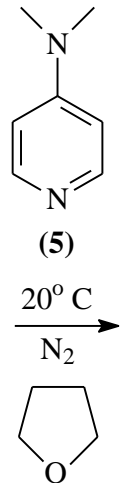

(4)

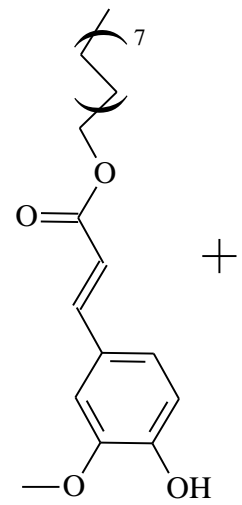

(6)

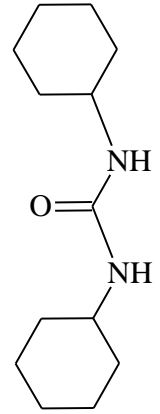

(7)

Figure 1. Scheme of Steglich esterification for preparing HF

In brief, $50 \mathrm{~mL}$ of THF was added to a round bottom flask. Then, $2.0 \mathrm{~g}$ $(10.3 \mathrm{mmoL})$ of FA and $12.48 \mathrm{~g}(51.5 \mathrm{mmoL})$ of $\mathrm{AC}$ were dissolved. Catalytic amounts of DMAP and $2.12 \mathrm{~g}(10.3 \mathrm{mmoL})$ of DCC were added to the previous solution. The atmosphere was replaced by nitrogen and the reaction mixture was kept under magnetic stirring and nitrogen flow for $12 \mathrm{~h}$ at $20^{\circ} \mathrm{C}$. After this time period, the reaction mixture was filtered twice using cotton for removing DCU. The final solution was then transferred to a round bottom flask for solvent removal using a rotary evaporator. This procedure was carried out from five independent batches.

\section{Purification of hexadecyl ferulate}

The final product was purified by column chromatography $(50 \mathrm{X} 500 \mathrm{~mm})$ filled with silica gel (230 mesh, 0.063-0.200 mm, Merck Millipore, Billerica, MA, USA) as the 
stationary phase at the silica:sample 10:1 (w:w) ratio. The elution was carried out by solvent passing using the hexane:ethyl acetate proportions with increasing order of polarity (100:0, 90:10, 80:20, 70:30, v:v) as mobile phase.

Thin layer chromatography (TLC) was used to analyze the fractions obtained by column chromatography. The silica gel 60 F254 (Merck Millipore, Billerica, MA, USA) and hexane:chloroform:ethyl acetate (70:20:10, v:v:v) were used as stationary and mobile phases, respectively. Vanillin and ferric chloride were used as colors reagents for detecting the spots. The substances under analysis (FA, CA and HF) were evidenced by the use of UV radiation at 254 and $366 \mathrm{~nm}$. The fractions containing a single spot on TLC plate were mixed and chosen for further analyses. These groups of fractions were dried for 2 days by freeze drying at $-40^{\circ} \mathrm{C}$ and $400 \mu \mathrm{Hg}$ vacuum. The final product $\mathrm{HF}$ was stored into a desiccator under vacuum at room temperature.

\section{Yield}

The gravimetric yield was determined by weighing the final product purified and by dividing this experimental value by the theoretical value calculated using stoichiometry. The results were expressed as mean \pm standard deviation (SD).

\section{Characterization of hexadecyl ferulate}

The ester product was characterized by spectroscopic methods in order to confirm its chemical structure.

Fourier-transformed infrared spectroscopy (FTIR)

The Fourier-transformed infrared spectra of FA, CA and HF were recorded from 4000 to $400 \mathrm{~cm}^{-1}$ on a Shimadzu IR Prestige-21 spectrophotometer (Kyoto, Japan) using $\mathrm{KBr}$ pellets with 32 scans and resolution of $4 \mathrm{~cm}^{-1}$.

Nuclear magnetic resonance (NMR) spectroscopy

For structural elucidation of $\mathrm{HF}$, the ${ }^{1} \mathrm{H}$ and ${ }^{13} \mathrm{C}$ NMR spectra were collected on a Bruker Avance DRX-400 instrument operated at $9.4 \mathrm{~T}$ for observing ${ }^{1} \mathrm{H}$ at 400.13 and ${ }^{13} \mathrm{C}$ at 100.61 . The TMS shift signal was used as the internal reference $(\delta=0 \mathrm{ppm})$. The spectrometer was equipped with a multinuclear direct detection $5 \mathrm{~mm}$ probe for recording ${ }^{1} \mathrm{H}$ and ${ }^{13} \mathrm{C}$ spectra. Deuterated chloroform was used for samples dilution.

\section{In vitro antioxidant potential}

In order to compare the antioxidant capacity of pure FA and HF, the antioxidant potential was evaluated by 2,2-diphenyl-1-picrylhydrazyl (DPPH•) radical scavenging assay and 2,2-azinobis(3-ethylbenzothiazoline-6-sulfonic acid) (ABTS) radical cation decolorization. Aqueous solutions of pure drug and $\mathrm{HF}\left(0.1\right.$ and $\left.50 \mu \mathrm{g} \cdot \mathrm{mL}^{-1}\right)$ were prepared $30 \mathrm{~min}$ before starting the experiments and kept under dark conditions.

\section{$\mathrm{DPPH} \cdot$ radical scavenging assay}

The antioxidant effect of the samples was tested against $60 \mu \mathrm{M}$ of the stable radical $\mathrm{DPPH} \bullet$ (2,2-diphenyl-1-picryl-hydrazyl) solubilized in absolute ethanol. The medium was composed by each sample in concentrations between 0.1 and $50 \mu \mathrm{g} . \mathrm{mL}^{-1}, \mathrm{DPPH}$ solution and absolute ethanol as reaction medium for obtaining $1000 \mu \mathrm{L}$. The mixture was incubated for 15 minutes at room temperature and protected from light. The scavenger action was observed by decreasing the absorbance at $\lambda=531 \mathrm{~nm}^{17}$.

ABTS radical cation decolorization assay 
The cationic radical ABTS•+ (2,2"-azinobis(3-ethylbenzothiazoline-6-sulfonic acid)) was prepared by reacting $5 \mathrm{~mL}$ of ABTS $\bullet+$ aqueous solution $(7 \mathrm{mM})$ with $88 \mu \mathrm{L}$ of persulfate of potassium $(2.46 \mathrm{mM})$ at room temperature, protected from light, for 12 to 16 hours prior to its use ${ }^{18}$. Prior to the assay, the ABTS $\bullet+$ solution was diluted with 10 $\mathrm{mM}$ sodium phosphate buffer (1:20 ratio). Samples at concentrations between 0.1 and $50 \mu \mathrm{g} \cdot \mathrm{mL}^{-1}$ were added to the plates together with ABTS•+ and $100 \mathrm{mM}$ sodium phosphate buffer in order to obtain a final volume of $1000 \mu \mathrm{L}$ of reaction medium. The reduction between ABTS $\bullet+$ and pure drug or HF was measured by decreasing the absorbance at $\lambda=734 \mathrm{~nm}$ after $15 \mathrm{~min}$.

All measurements were performed in triplicate using a microplate reader (SpectraMax 190 spectrophotometer, Molecular Devices, Sunnyvale, CA, USA). Quercetin ( $\geq 95 \%$ ) was used as positive control. The antioxidant activity was calculated as percentage of inhibition according to the Equation 1.

$\%$ Inhibition $=\left[\frac{(A b-A a)}{A b}\right] \times 100$

Where $A b$ is the absorbance of the control and $A a$ is the absorbance of the sample.

Statistical analysis

Data were expressed as mean \pm standard deviation (SD) using Graph Pad Prism version 6.0 for windows, Graph Pad Software (San Diego, CA, USA). Statistical analysis was performed by ANOVA followed by Bonferroni's post-hoc test and a $p$ value lower than 0.05 was considered as statistically significant. The $50 \%$ inhibitory concentration (IC50) was calculated from the dose response curve (Graph Pad Prism Version 6.0) obtained by plotting percentage inhibition versus concentrations.

\section{RESULTS AND DISCUSSION}

\section{Synthesis of hexadecyl ferulate}

FH was successfully obtained as an off-white powder by the Steglich esterification. This process provided a mild reaction medium since it was kept at low temperature under an inert atmosphere ${ }^{13}$. In addition, the resulting water reacted with DCC for obtaining the by-product DCU which avoided the ester hydrolysis by water.

\section{Purification of hexadecyl ferulate}

Two bands were observed during purification which suggested the separation of two fractions when the eluent hexane:ethyl acetate at 80:20 proportion passed through the column. HF was the first compound purified. It was detected by UV and confirmed by TLC with a retention factor of 0.69 . After solvents' evaporation, HF was obtained as a white powder.

\section{Yield}

A yield of $76.77 \pm 1.35 \%$ was obtained after synthesis and purification of hexadecyl ferulate. This yield is in accordance with those reported in literature for esterification reactions ${ }^{13}$. Bernards and Lewis (1992) ${ }^{19}$ obtained yields lower than $25 \%$ and $66 \%$ using DCC and DCC after the addition of a labile acid as a shielding reagent to the free FA hydroxyl group, respectively. Another study, which carried out the esterification of FA by means of biocatalysts, reached yields between 44 and $56 \%{ }^{20}$. These results reinforce the efficacy of the Steglich esterification for preparing HF using DCC and DMAP as a catalytic agent.

\section{Characterization of hexadecyl ferulate}


Fourier-transformed infrared spectroscopy (FTIR)

FTIR spectra performed for FA, CA and HF are shown in Figure 2. The FTIR spectrum for pure FA consisted of absorption bands of $\mathrm{OH}$ group stretching vibration $\left(3441 \mathrm{~cm}^{-1}\right)$, aromatic and alkene $\mathrm{C}-\mathrm{H}$ stretching vibrations $\left(3018 \mathrm{~cm}^{-1}\right)$, alkane $\mathrm{C}-\mathrm{H}$ stretching vibration $\left(2974 \mathrm{~cm}^{-1}\right)$, aromatic conjugated $\mathrm{C}=\mathrm{O}$ stretching vibration (1691 $\left.\mathrm{cm}^{-1}\right), \mathrm{C}=\mathrm{C}$ aromatic stretching vibration $\left(1620,1598,1516,1431 \mathrm{~cm}^{-1}\right), \mathrm{C}-\mathrm{O}-\mathrm{C}$ asymmetric stretching vibration $\left(1273 \mathrm{~cm}^{-1}\right)$, and $\mathrm{C}-\mathrm{OH}$ stretching vibration $\left(1205 \mathrm{~cm}^{-}\right.$ $\left.{ }^{1}\right)$. Concerning CA, its infrared spectrum exhibited two bands at 2850 and $2920 \mathrm{~cm}^{-1}$ due to symmetric and asymmetric $-\mathrm{CH}_{2}$ stretching, respectively. A broad band assigned to $-\mathrm{OH}$ stretching was observed between 3360 and $3236 \mathrm{~cm}^{-1}$. HF showed a FTIR spectrum presenting two typical bands of ester group (Figure 2). HF demonstrated a $\mathrm{C}=\mathrm{O}$ ester stretching band at $1725 \mathrm{~cm}^{-1}(\boldsymbol{a})$ and a $\mathrm{C}-\mathrm{O}$ stretching band at $1159 \mathrm{~cm}^{-1}(\boldsymbol{b})$ which confirmed that FA ester was obtained. Moreover, the broad band between 3360 and $3236 \mathrm{~cm}^{-1}$ (c) assigned to $-\mathrm{OH}$ stretching of CA was not observed in HF which suggested that this hydroxyl group was consumed for ester synthesis. Therefore, these FTIR assignments suggest that the synthesis of FA ester was successfully performed by the previously described Steglich esterification.

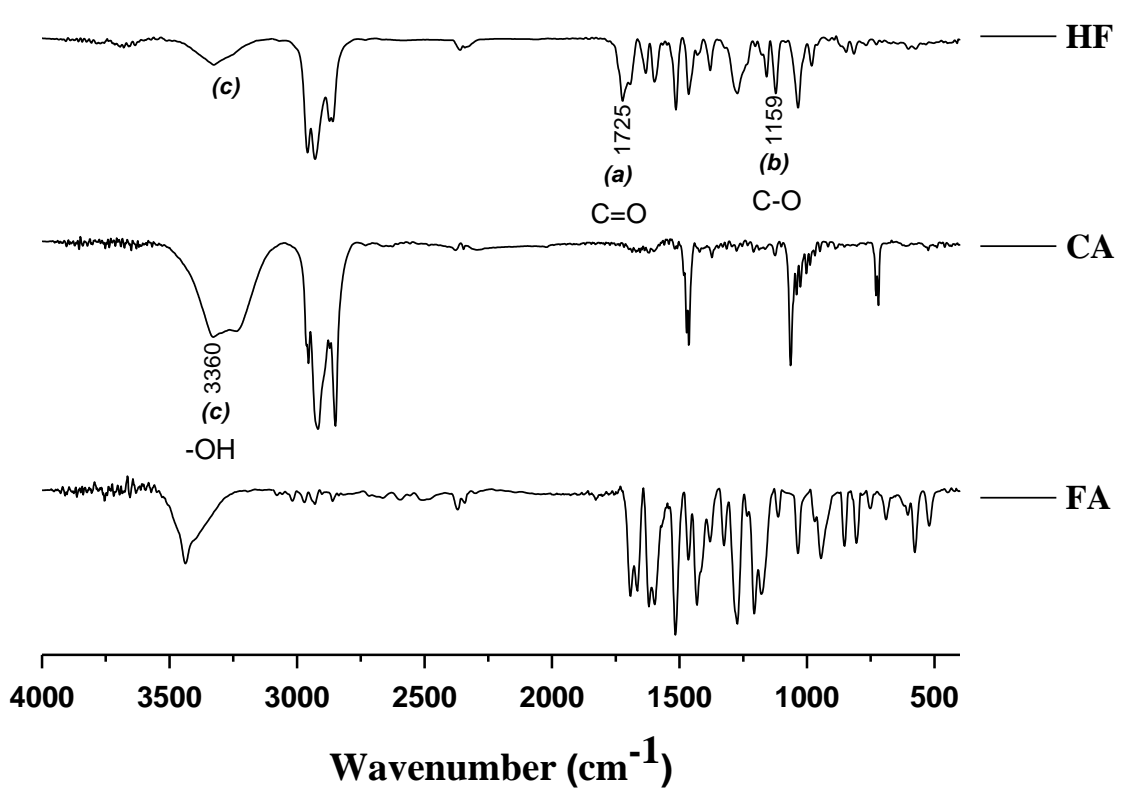

Figure 2. FTIR spectra of ferulic acid (FA), cetyl alcohol (CA) and hexadecyl ferulate (HF).

\section{Nuclear Magnetic Resonance Spectroscopy (NMR)}

The ${ }^{1} \mathrm{H}$ and ${ }^{13} \mathrm{C}$ NMR spectral data confirmed the chemical structure of $\mathrm{HF}$. Considering ${ }^{1} \mathrm{H}$ NMR spectrum of HF (Figure 3 ), ${ }^{1} \mathrm{H}$ chemical shifts of the protons of the aromatic ring were assigned at $\delta 7.08(\boldsymbol{i}), 7.05(\boldsymbol{l})$ and $6.94(\boldsymbol{m}) \mathrm{ppm}$ and a shift signal at $\delta 4.22(\boldsymbol{d})$ ppm corresponded to the hydrogens of the first carbon in the ester alkyl chain which suggested that the ester group was obtained. Besides, the other signals of chemical shifts between $\delta 1.72$ and $0.88(\boldsymbol{a}, \boldsymbol{c}) \mathrm{ppm}$ were assigned to the hydrogens of the other carbons in the alkyl chain. Regarding the 13C NMR spectrum (Figure 4), HF showed a chemical shift at $\delta 167.39$ (e) ppm which corresponded to the carbonyl carbon of the ester group and confirmed the synthesis of FA ester. 


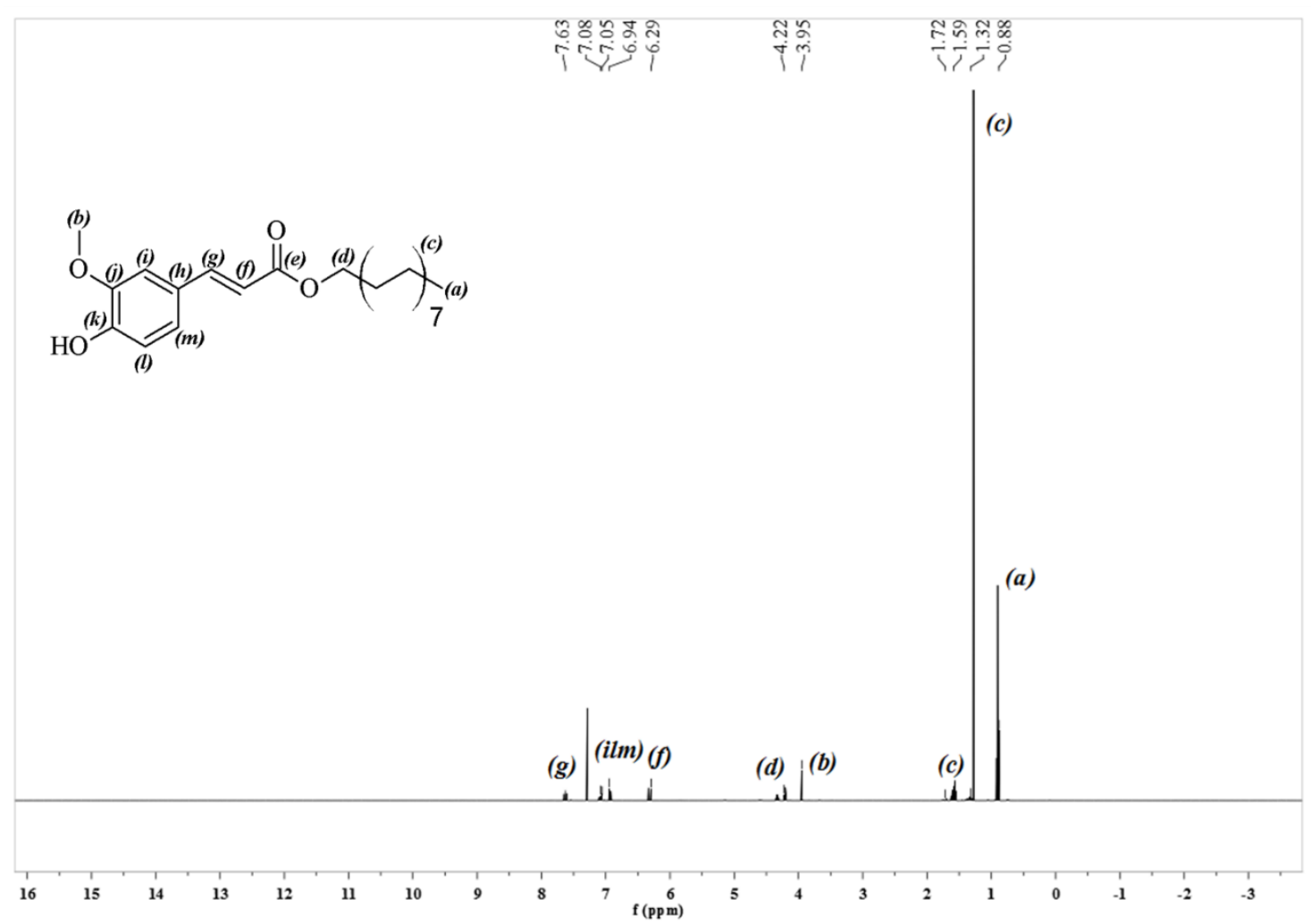

Figure 3. ${ }^{1} \mathrm{H}$ NMR spectrum of HF.

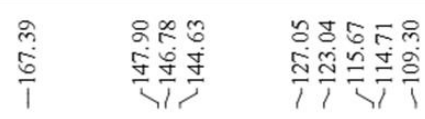<smiles>[3H]C([3H])([3H])CCC(C)(C)OC(=O)/C=C/c1ccc(O)c(O[Hg])c1</smiles>

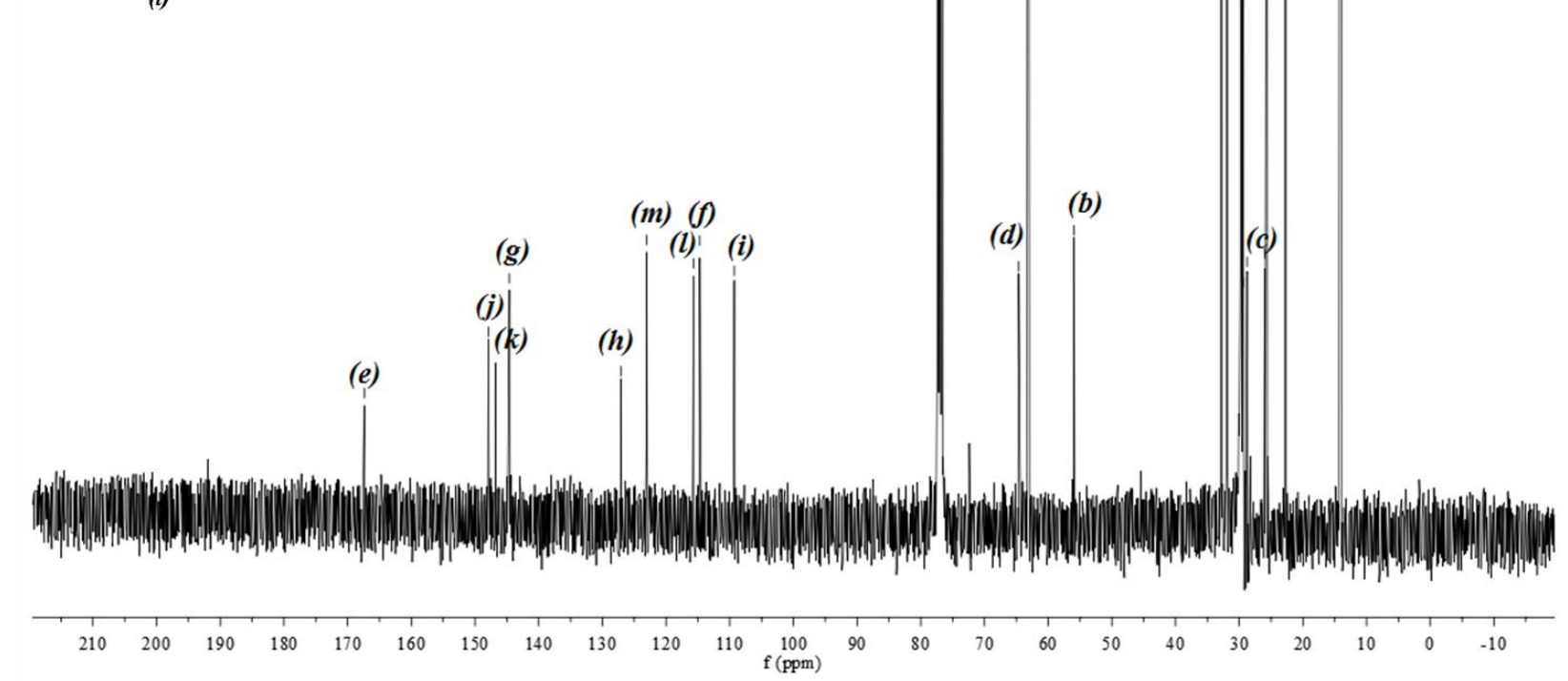

Figure 4. ${ }^{13} \mathrm{C}$ NMR spectrum of HF. 
In vitro antioxidant potential

In order to explore whether the structural modification has influence on antioxidant capacity, the profile of action of FA and HF on the free radical DPPH $\bullet$ and the reactive oxygen species ABTS $\bullet+$ were compared at different concentrations.

$D P P H \cdot$ radical scavenging assay

$\mathrm{DPPH} \bullet$ is a stable radical widely used in antioxidant trials in the field of organic compounds and natural products. HF proved its antioxidant potential leading to a half maximal inhibitory concentration (IC50) of $0.083 \pm 0.009 \mathrm{nmol} \cdot \mathrm{mL}^{-1}$ while FA achieved a higher IC50 value of $0.160 \pm 0.010 \mathrm{nmol} \cdot \mathrm{mL}^{-1}$. Therefore, HF reached a scavenger activity in statistically lower concentrations than FA which differs that reported by Anselmi et al. (2004) ${ }^{14}$. These authors investigated the scavenger activity of some different alkyl esters of FA on DPPH • radical scavenging assay and showed no significant differences between FA and its derivatives compounds. Quercitin is a well-known antioxidant standard ${ }^{21}$ and showed an IC50 of $0.030 \pm 0.001 \mathrm{nmol} . \mathrm{mL}^{-1}$. This flavonoid presented an improved scavenger activity that was statistically different than HF and FA.

ABTS radical cation decolorization assay

The cationic radical of ABTS $\bullet+$ is a synthetic nitrogen-substituted aromatic compound widely used to evaluate the abilities of phenolic agents as radical scavengers ${ }^{18}$. Considering their $\%$ of inhibition (Figuree 5), HF $\left(99.74 \pm 4.68 \%\right.$ at $0.05 \mathrm{nmol}^{-\mathrm{mL}^{-1}}$ ), FA $\left(98.41 \pm 0.53 \%\right.$ at $\left.0.01 \mathrm{nmol} . \mathrm{mL}^{-1}\right)$ and quercitin $(98.66 \pm 0.33 \%$ at 0.007 nmol.mL $\mathrm{mL}^{-1}$ ) showed no statistically significant difference on scavenging activity as previously reported Menezes et al. (2011) ${ }^{22}$. In spite of HF showed the higher \% of inhibition, this FA ester achieved an IC50 value of $0.027 \pm 0.002 \mathrm{nmol} . \mathrm{mL}^{-1}$ while FA and quercitin demonstrated $50 \%$ of inhibition at lower concentrations of $0.005 \pm 0.001$ and $0.002 \pm 0.001 \mathrm{nmol} . \mathrm{mL}^{-1}$, respectively. This could be justified by the spatial conformation, arrangement and reaction behavior of HF after Steglich esterification ${ }^{14}$.

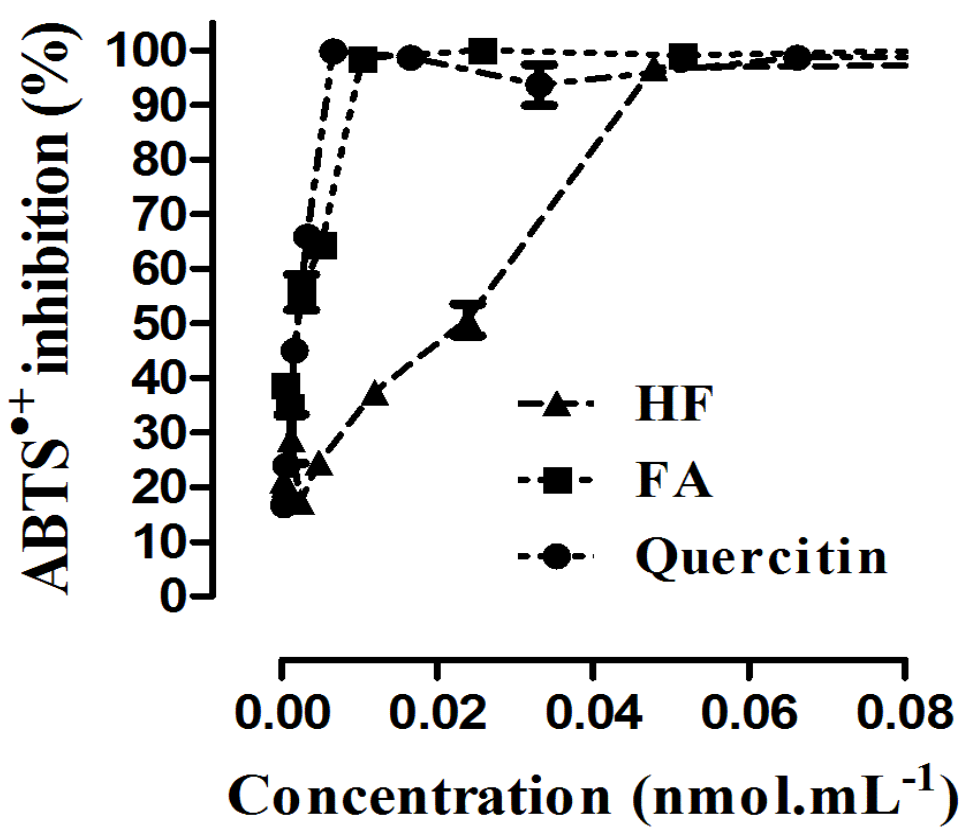

Figure 5. Antioxidant effect of ferulic acid (FA), hexadecyl ferulate (HF) and quercetin by ABTS•+ radical cation decolorization assay. 
In addition, further studies are required in order to investigate the antioxidant effect of $\mathrm{HF}$ in biological systems. According to literature ${ }^{14}$, HF makes it less susceptible to interacting, through van der Walls, with the phospholipids of membrane and remains anchored therein when compared to other alkyl esters ferulic acid derivatives. Comparing the partition coefficient $(\log \mathrm{P})$ values of HF and FA (8.86 and 1.25, respectively), HF would be more susceptible to $\mathrm{BBB}$ transpose due to a close relationship between the $\log \mathrm{P}$ of a drug and its permeability in $\mathrm{BBB}^{15}$. In addition, some reports suggest that the compound solubility in lipids can be an important factor for the suppression efficiency of lipid oxidation and that alkyl chain ferulates would be more promising antioxidants than $\mathrm{FA}^{16}$.

\section{CONCLUSION}

The synthesis of HF was successfully performed from FA by Steglich esterification. This FA ester was confirmed by FTIR and $1 \mathrm{H}$ and 13C NMR spectra. HF demonstrated an equivalent or improved antioxidant potential than FA by the free radical $\mathrm{DPPH} \bullet$ and reactive oxygen species ABTS •+ assays.

\section{ACKNOWLEDGEMENTS}

The authors thank to Conselho Nacional de Pesquisa e Desenvolvimento (CNPq) for financial support (grant number 456403/2014-0). The authors have no direct relation or financial involvement with any commercial organization with interest in the subject or materials discussed in this paper.

\section{REFERENCES}

1. Nagai N, Kotani S, Mano Y, Ueno A, Ito Y, Kitaba T, Takata T, Fujii N. Ferulic acid suppresses amyloid $\beta$ production in the human lens epithelial cell stimulated with hydrogen peroxide. BioMed Res. Int. 2017; 2017: 1-9.

2. Kikugawa M, Tsuchiyama M, Kai K, Sakamoto T. Synthesis of highly water-soluble feruloyl diglycerols by esterification of an Aspergillus niger feruloyl esterase. Appl Microbiol Biotechnol. 2012; 95: 615-622.

3. Antonopoulou I, Leonov L, Jütten P, Cerullo G, Faraco V, Papadopoulou A, Kletsas D, Ralli M, Rova U, Christakopoulos P. Optimized synthesis of novel prenyl ferulate performed by feruloyl esterases from Myceliophthora thermophila in microemulsions. Appl Microbiol Biotechnol. 2017; 101: 3213-3226.

4. Nadal JM, Gomes MLS, Borsato DM, Almeida MA, Barboza FM, Zawadzki SF, Kanunfre CC, Farago PV, Zanin SMW. Spray-dried Eudragit ${ }^{\circledR}$ L100 microparticles containing ferulic acid: Formulation, in vitro cytoprotection and in vivo anti-platelet effect. MSEC. 2016; 64: 318-328.

5. Schroeter H, Williams RJ, Matin R, Iversen L, Rice-Evans CA. Phenolic antioxidants attenuate neuronal cell death following uptake of oxidized low-density lipoprotein. Free Radic Biol Med. 2000; 29(12): 1222-1233.

6. Tan Z, Shahidi F. Chemoenzymatic synthesis of phytosteryl ferulates and evaluation of their antioxidant activity. J. Agric. Food Chem. 2011; 59: 12375-12383.

7. Nadal JM, Gomes MLS, Borsato DM, Almeida MA, Barboza FM, Zawadzki SF, Farago PV, Zanin SMW. Spray-dried solid dispersions containing ferulic acid: comparative analysis of three carriers, in vitro dissolution, antioxidant potential and in vivo anti-platelet effect. Drug Dev Ind Pharm. 2016; 42(11): 1813-1824.

8. Mancuso C, Santangelo R. Ferulic acid: Pharmacological and toxicological aspects. Food Chem Toxicol. 2014; 65: 185-195.

9. Wu K, Wang Z-Z, Liu D, Qi X-R. Pharmacokinetics, brain distribution, release and bloodbrain barrier transport of Shunaoxin pills. J Ethnopharmacol. 2014; 151: 1133-1140. 
10. Alyautdin R, Khalin I, Nafeeza MI, Haron MH, Kuznetsov D. Nanoscale drug delivery systems and the blood-brain barrier. Int. J. Nanomed. 2014; 9: 795-811.

11. Huang K-C, Li Y, Kuo C-H, Twu Y-K, Shieh C-J. Highly Efficient Synthesis of an Emerging Lipophilic Antioxidant: 2-Ethylhexyl Ferulate. Molecules 2016; 21,478: 1-11.

12. Sandoval G, Quintana PG, Baldessari A, Ballesteros AO, Plou FJ. Lipase-catalyzed preparation of mono- and diesters of ferulic acid. Biocatal Biotransformation. 2015; 33(2): 89-97.

13. Neises B, Steglich W. Simple Method for the Esterification of Carboxylic Acids. Angew. Chem. Int. Ed. 1978; 17(7): 522-524.

14. Anselmi C, Centini M, Granata P, Sega A, Buonocore A, Bernini A, Facino RM. Antioxidant activity of ferulic acid alkyl esters in a heterophasic system: a mechanistic insight. J Agric Food Chem. 2004; 52(21): 6425-6432.

15. Lipinski CA, Lombardo F, Dominy BW, Feeney PJ. Experimental and computational approaches to estimate solubility and permeability in drug discovery and development settings. Adv Drug Deliv Rev. 2001; 46(1-3): 3-26.

16. Fang X, Shima M, Kadota M, Tsuno T, Adachi S. Suppressive effect of alkyl ferulate on the oxidation of linoleic acid. Biosci Biotechnol Biochem. 2006; Feb;70(2): 457-461.

17. Mihaylova D, Schalow S. Antioxidant and stabilization activity of a quercetin containing flavonoid extract obtained from bulgarian Sophora japonica L. Braz. Arch. Biol. Technol. 2013; 56(3): 431-438.

18. Re R, Pellegrini N, Proteggente A, Pannala A, Yang M, Rice-Evans C. Antioxidant activity applying an improved ABTS radical cation decolorization assay. Free Radic Biol Med. 1999; 26(9/10):1231-1237.

19. Bernards MA, Lewis NG. Alkyl ferulates in wound healing potato tubers. Phytochem.1992; 31(10): 3409-3412.

20. Chigorimbo-Murefu NTL, Riva S, Burton SG. Lipase-catalysed synthesis of esters of ferulic acid with natural compounds and evaluation of their antioxidant properties. J. Mol. Catal., B Enzym. 2009; 56(4): 277-282.

21. D'Andrea G. Quercetin: A flavonol with multifaceted therapeutic applications? Fitoterapia. 2015; 106: 256-271.

22. Menezes JC, Kamat SP, Cavaleiro JA, Gaspar A, Garrido J, Borges F. Synthesis and antioxidant activity of long chain alkyl hydroxycinnamates. Eur J Med Chem. 2011; 46(2):773-777. 